\title{
Female sex hormones and the recovery from exercise: Menstrual cycle phase affects responses
}

\author{
Anthony C. Hackney ${ }^{1}$, Ashley L. Kallman ${ }^{1}$, Eser Ağgön ${ }^{2}$ \\ ${ }^{1}$ Department of Exercise \& Sport Science, University of North Carolina, Chapel Hill, North Carolina, USA; ${ }^{2}$ College of \\ Physical Education and Sport, Erzincan University, Erzincan, Turkey
}

\section{Summary}

Study aim: Evidence supports female sex hormones have an influencing effect on a multitude of physiological and psychological systems related to exercise. Little is known, however, whether is effect persist into the recovery from exercise. Our objective was to examine aspects of muscle damage/inflammation process during recovery in healthy, exercise-trained women following endurance activity at the mid-follicular (MF; low sex hormone level) and mid-luteal (ML; elevated sex hormone levels) phases of their menstrual cycle.

Material and methods: The MF and ML exercise sessions consisted of running for 90 minutes at $70 \% \mathrm{VO}_{2 \max }$ on a treadmill in a controlled laboratory environment. Menstrual cycle phase was hormonally confirmed, diet and physical activity was control throughout the study. Outcome measures were: blood creatine kinase (CK) and interleukin-6 (IL-6) assessed at immediate-post exercise (IP), 24-hour and 72-hour into recovery. Statistics involved ANOVA procedures.

Results: At 24-hours and 72-hour into recovery CK activity was greater in MF than ML $(p<0.05)$ while for IL-6 at IP, 24-hour and 72-hour responses were significantly greater at MF than at ML $(p<0.05)$.

Conclusions: A more robust recovery CK and IL-6 response occur in the MF of the menstrual cycle when female sex hormones are reduced. This finding suggests female sex hormone changes due to menstrual cycle phase affect the physiologic responses during the extended recovery period from intensive exercise in eumenorrheic women.

Key words: Estrogen - Creatine kinase - Cytokines - IL-6 - Exercise training

\section{Introduction}

Over the last few decades, there has been an exponential growth in the number of women engaging in exercise and exercise training programs. However, the amount of exercise research performed studying women has remained relatively limited compared to that of men. This has occurred, in part, because of the difficult nature of controlling for the hormonal fluctuations associated with the menstrual cycle of women [1]. Specifically, the female sex hormones, estrogen, and progesterone, secreted primarily from the ovaries follow a distinct pattern of large cyclic changes in eumenorrheic women. Although sex hormones function primarily to support reproduction, evidence supports an effect on a multitude of other physiological and psychological systems which impacts on exercise responses and adaptations $[2,3]$.
Interestingly, most research studies examining the effect of the menstrual cycle (MC) hormonal fluctuations on exercise have focused upon what happens during an exercise session or at the immediate post-recovery from exercise. Many aspects of the effect of exercise on physiology, however, occur during the extended post-exercise recovery time, which is also a critical period relative to the adaptation process associated with training regimes [4]. Anecdotally our research group has observed a greater variance in a variety of biomarkers response during the extended recovery period of women athletes. This caused us to wonder what role female sex hormones changes across the MC might be having on such a recovery process. To address this, we conducted a pilot study to systematically examine aspects of the recovery from intensive exercise in healthy, exercise-trained women. Specifically, for this experiment we had women perform 90 minutes of running exercise at the mid-follicular (MF; low sex hormone level)

Author's address Professor Anthony C. Hackney, University of North Carolina, CB \# 8700 - Fetzer Hall, Chapel Hill, NC USA 27599 ach@email.unc.edu 
and mid-luteal (ML; elevated sex hormone levels) phases of their $\mathrm{MC}$ and examined select physiological responses throughout 72 hours of recovery from the exercise.

\section{Material and methods}

Eight trained female runners were recruited for this study. All participants had: normal menstrual cycles over the last year, competed regularly in road-running competitions, sustained no major injuries with the last six months, exercise-trained a minimum of 4-5 days per week, and performed 45 to 120 minutes per exercise session/day aerobically within their training. Their physical characteristics were: age $=25 \pm 4$ years, height $=167.8 \pm 3.1 \mathrm{~cm}$, body mass $=58.9 \pm 3.7$, body fat $=22.5 \pm 4.4 \%$, and $\mathrm{VO}_{2 \max }=57.5 \pm 3.5 \mathrm{ml} \cdot \mathrm{kg}^{-1} \cdot \mathrm{min}^{-1}$.

The subject's MC length was monitored for six months prior to the study to ensure a eumenorrheic state. The average MC length (mean \pm SD) was $28 \pm 3$ days. The MF phase exercise session occurred at $8 \pm 2$ days after the onset of menses and the ML session was at 23 \pm 3 days after the onset of menses. Resting hormonal analysis of blood samples for $17 \beta$-estradiol $\left(\mathrm{E}_{2}\right)$ and progesterone confirmed the appropriate menstrual phase and hormonal condition. A diet moderately high in carbohydrates ( $\sim 55 \%$ of daily caloric intake) was consumed during the 24-hours prior to both the MF and ML exercise sessions, and no exercise was performed during this 24-hour period to ensure the subjects were rested and well hydrated.

The MF and ML exercise sessions consisted of running for 90 minutes at $70 \%$ maximal oxygen uptake $\left(\mathrm{VO}_{2 \max }\right)$ on a treadmill in a controlled laboratory environment, administered in random order. Running speed was $13.0 \pm 0.5 \mathrm{~km} \cdot \mathrm{h}^{-1}$. Table 1 shows select physiologic responses during the exercise and illustrates the exercise sessions were demanding, but similar at MF and ML.

Since this was an unfunded pilot study, only limited biological measurements at select time points were made during the extended post-exercise recovery period (i.e.,
72 hours) - specifically directed towards the inflammation process (Interleukin-6 [IL-6]) and muscle damage (creatine kinase [CK]) (Abnova Corporation, Taiwan) [5, 7]. Throughout the days of recovery, subjects did no exercise training, ate an iso-caloric diet and consumed fluid ad libitum.

Data statistical analysis involved descriptive and inferential procedures. The latter employing the Friedman ANOVA and the Nemenyi post hoc procedure [6]. The alpha level was set at 0.05 .

\section{Results}

Resting, immediate-post exercise (IP), 24-hour and 72-hour into recovery, CK activity, and IL-6 concentration responses are reported in Table 2. Statistical analysis indicated a significant effect for cycle phase and time $(p<0.05)$ for both CK and IL-6.

CK activity at rest and at IP was not significantly different between phases $(p>0.05)$. However, at 24-hours and 72-hour following the prolonged running bout, $\mathrm{CK}$ activity was greater in MF than ML $(p<0.05)$. IL-6 was not significantly different at rest in the MF and ML sessions $(p>0.05)$, but the IP, 24-hour and 72-hour responses were significantly greater at MF than at ML $(p<0.05)$.

\section{Discussion}

These findings demonstrate that during the extended recovery from prolonged, intensive endurance-based exercise blood parameters of CK and IL-6 vary considerably, based upon the phase of the $\mathrm{MC}$ in women.

The biomarker CK is commonly used as a representation of muscle damage since its principally found in muscle tissue [7], while IL-6 is a pleiotropic cytokine that can be reflective of the pro-inflammation process [4]. That is, specifically, IL-6 plays a key role in the acute phase response of the inflammation process by facilitating the

Table 1. Mean $( \pm \mathrm{SD})$ of heart rate (HR) and Borg rating of perceived exertion (RPE) during prolonged running bouts during MF and ML phases of the menstrual cycle (MF and ML responses, $p>0.05$ )

\begin{tabular}{llccccc}
\hline \multirow{2}{*}{ Phase } & Measure & \multicolumn{5}{c}{ Time [minutes] } \\
\cline { 3 - 7 } & & Rest & 10 & 30 & 60 & 90 \\
\hline \multirow{2}{*}{ Mid-follicular (MF) } & HR (bpm) & $60 \pm 8$ & $156 \pm 10$ & $161 \pm 7$ & $165 \pm 6$ & $170 \pm 6$ \\
& RPE & 6 & $12 \pm 1$ & $12 \pm 1$ & $13 \pm 1$ & $14 \pm 1$ \\
\multirow{2}{*}{ Mid-luteal (ML) } & HR (bpm) & $67 \pm 6$ & $153 \pm 5$ & $159 \pm 5$ & $162 \pm 4$ & $170 \pm 5$ \\
& RPE & 6 & $10 \pm 1$ & $13 \pm 1$ & $13 \pm 1$ & $14 \pm 1$ \\
\hline
\end{tabular}


Table 2. Mean ( $\pm \mathrm{SD}$ ) blood creatine kinase (CK) activity and interleukin-6 (IL-6) concentration at rest and in response to prolonged running bouts during MF and ML phases of the menstrual cycle

\begin{tabular}{lcccc}
\hline CK $[\mathrm{U} / \mathrm{L}]$ & Rest & IP & 24-hour & 72-hour \\
\hline MF & $89.7 \pm 16.7$ & $109.7 \pm 10.7$ & $510.8^{*} \pm 344.9$ & $425.7^{*} \pm 249.7$ \\
ML & $92.6 \pm 9.6$ & $112.7 \pm 16.8$ & $275.1 \pm 55.1$ & $211.2 \pm 23.4$ \\
\hline IL-6 [pg/mL] & & & & \\
\hline MF & $1.4 \pm 1.9$ & $24.9^{*} \pm 13.2$ & $10.3 * \pm 7.1$ & $4.3^{*} \pm 3.1$ \\
ML & $1.2 \pm 0.5$ & $13.5 \pm 6.2$ & $5.0 \pm 3.0$ & $0.9 \pm 0.3$ \\
\hline
\end{tabular}

* denotes significant differences between MF and ML values.

production of acute phase proteins. IL-6 in combination with its soluble receptor sIL-6R $\alpha$ dictates the transition from acute to chronic inflammation by changing the nature of leucocyte infiltrate (i.e., polymorphonuclear neutrophils to monocyte/macrophages) [4]. The responses of these key parameter are part of the adaptive and recovery process in response to exercise training sessions [5]; but, notably, a multitude of others also exist and are involved with this process.

Our present pilot study is limited in sample size, measurement parameters, and measurement sampling time/frequency. Nonetheless, even with these limitations, the current data support that MC phase and the associated sex hormone fluctuations do affect physiological aspects of the recovery process from exercise in women - specifically, a more robust CK and IL-6 response occurs in the MF of the menstrual cycle when female sex hormones are reduced. These biomarker findings could signal the need for a greater recovery period from intensive endurance exercise in the MF phase of the cycle, perhaps due to the anti-oxidant aspects of estrogens being mitigated since hormonal concentrations are reduced at this time [7]. Although we acknowledge this is speculation on our part.

In conclusion, the current evidence strongly suggests that exercise scientists should pursue studying how female sex hormone fluctuations due to MC cycle phase affect the physiologic responses during the extended recovery period from exercise in eumenorrheic women.

Conflict of interest: Authors state no conflict of interest.

\section{References}

1. Bruinvels G., Burden R.J., McGregor A.J., Ackerman K.E., Dooley M., Richards T., Pedlar C. (2017) Sport, exercise and the menstrual cycle: where is the research? Br. J. Sports Med., 51: 487-488.

2. Chrousos G.P., Torpy D.J., Gold P.W. (1998) Interactions between the Hypothalamic-Pituitary-Adrenal Axis and the Female Reproductive System: Clinical Implications. Ann. Intern. Med., 129(3): 229-240.

3. Davis H., Hackney A.C. (2017) The hypothalamic-pituitary-ovarian axis and oral contraceptives: regulation and function. In: Hackney AC (ed.), Sex Hormones, Exercise and Women. Springer International Publishing, pp. 1-18.

4. Gabay C. (2006) Interleukin-6 and chronic inflammation. Arthritis Res. Ther., 8(Suppl 2): S3.

5. Kenny W.L., Wilmore J.H., Costill D.L. (2017) Physiology of Sport and Exercise. $6^{\text {th }}$ Edition. Human Kinetics Publishing.

6. Linton M., Gallo P.S. (1975) The Practical Statistician: Simplified Handbook of Statistics. Brooks/Cole Publishing Co., pp. 115-120, 311-315.

7. Tiidus P.M. (2017) Estrogen and menopause: Muscle damage, repair and function in females. In: Hackney AC (ed.), Sex Hormones, Exercise and Women. Springer International Publishing, pp. 71-86.

\section{Received 07.03.2019 \\ Accepted 07.05.2019}

(C) University of Physical Education, Warsaw, Poland 